\title{
Fístula traqueoesofágica, a propósito de un caso
}

\section{Tracheoesophageal fistula, a case report}

\author{
Luz Alejandra Vargas Espíndola ${ }^{1}$, Ana Milena Callejas ${ }^{2}$, Alfredo
} Saavedra ${ }^{3}$, Edgar Alberto Sánchez Morales Rodríguez ${ }^{4}$

\section{Resumen}

La fístula traqueoesofágica es una patología infrecuente que en general se asocia con patología tumoral esofágica y algunas veces con tumores primarios de vía aérea, que implican pobre sobrevida y mal pronóstico a corto plazo. El tratamiento requiere un enfoque multidisciplinario, se considera la colocación de endoprótesis como manejo de primera línea para alivio de síntomas y mejoría de calidad de vida. El artículo reporta el caso de un paciente con carcinoma escamocelular de tercio medio del esófago inoperable, con colocación exitosa de doble stent esofágico y traqueal.

Palabras clave: Fístula traqueoesofágica; fístula traqueoesofágica maligna; procedimiento quirúrgico endoscópico; stent vía aérea; stents metálicos autoexpandibles; terapia paliativa.

\begin{abstract}
Tracheoesophageal fistula is a rare condition that is generally associated with esophageal cancer and sometimes primary airway cancer and involves poor survival and short-term prognosis. Treatment requires a multidisciplinary approach and stenting is considered first line management for symptom relief and improved quality of life. The article reports the case of a patient with inoperable squamous cell carcinoma of the middle third of the esophagus with successful placement of double esophageal and tracheal stents.
\end{abstract}

Keywords: Tracheoesophageal fistula; malignant tracheoesophageal fistula; endoscopy; airway stenting; self-expandable metallic stents; palliative care.

\footnotetext{
${ }^{1}$ Enfermera y Médica-Cirujana. Facultad de Medicina, Universidad Nacional de Colombia. https://orcid.org/00000002-9472-6181

2 Médica neumóloga. Internista especializada en la atención de pacientes respiratorios en los ámbitos hospitalarios y ambulatorios, con énfasis en pacientes oncológicos de alta complejidad. Instituto Nacional de Cancerología.

${ }^{3}$ Médico especialista en Medicina Interna Universidad El Bosque. Médico Instituto Nacional de Cancerología.

${ }^{4}$ Médico especialista en Medicina Interna Universidad El Bosque. Médico Instituto Nacional de Cancerología.
}
Autor de Correspondencia Luz Alejandra Vargas Espíndola Correo electrónico: lavargase@unal.edu.co

Recibido: $26 / 01 / 2021$

Aceptado: 06/10/2021 


\section{Introducción}

Las fístulas traqueoesofágicas (FTE) son comunicaciones anormales entre el esófago y la tráquea o bronquio principal, denominándose fistulas broncoesofágicas, las cuales comparten un abordaje diagnóstico y terapéutico similar.

Las FTE pueden ser congénitas o adquiridas, pueden ocurrir después de cirugía, radioterapia, quimioterapia o por invasión de la vía aérea. La mayoría de las FTE en los adultos son adquiridas, siendo la mitad secundarias a malignidad esofágica o pulmonar $(1,2)$. Cuando una FTE maligna se desarrolla en cáncer primario, si es de esófago o tráquea es invariablemente incurable (3). La mayoría de las fístulas tienen una localización esofagotraqueal en casi un $57 \%$, esofagobronquial en un $40 \%$ y el restante con una disposición esofagopulmonar (4). Los pacientes con FTE tienen mala calidad de vida y un pobre pronóstico con sobrevida medida en días a semanas con FTE sin intervención y en meses, después de colocación de stent (5). Se debe sospechar la F'TE por la presencia de tos con la ingestión de líquidos o sólidos, bronquitis, neumonía recurrente y malnutrición. El diagnóstico incluye la combinación de hallazgos clínicos, imagenológicos y endoscópicos que demuestren fuga o la FTE.

El tratamiento requiere un enfoque multidisciplinar y será quirúrgico o endoscópico teniendo en cuenta la etiología, el tamaño, la anatomía de la FTE y las comorbilidades del paciente, así como el pronóstico de su enfermedad (6).

El propósito de este artículo es presentar el reporte de caso de una F'TE maligna en un paciente de 62 años y revisar la literatura enfocada en el tema y las opciones de manejo endoscópico.

\section{Reporte de caso}

Paciente masculino de 62 años exfumador pesado, con enfermedad pulmonar obstructiva crónica (EPOC) dependiente de oxígeno, con clasificación de severidad GOLD (estrategia mundial para la enfermedad pulmonar obstructiva crónica) C. Presenta antecedente de carcinoma escamocelular de tercio medio del esófago estadio cT4bN+M0, localmente avanzado en manejo con radioterapia y quimioterapia paliativa, por presentar signos de irresecabilidad quirúrgica. Ingresa por cuadro clínico de una semana de evolución consistente en tos seca que aumentaba con el consumo de alimentos asociada a disfagia para líquidos y sólidos, pérdida de peso de 3 kilos. Se realizó esófagograma que mostró paso de medio de contraste a la vía aérea. En la endoscopia de vías digestivas altas se evidenció área de mucosa friable, edematizada con fibrina, que comprometía aproximadamente el $40 \%$ de la circunferencia del esófago con umbilicación central compatible con fístula, con mucosa de aspecto neoplásico y presencia de estenosis.

Se realizó fibrobroncoscopia que mostró a 3 centímetros de la glotis compresión extrínseca de la pared posterior de la tráquea, con infiltración de la mucosa traqueal en una extensión de 3 centímetros (Figura 1). Dados los hallazgos, se programa colocación de stent traqueal y esofágico. Se coloca a nivel de la tráquea stent ultraflex autoexpandible recubierto de 80 por 20 milímetros, sin complicaciones (Figura 2). En la radiografía de tórax (Figura 3) se evidencia adecuado posicionamiento de endoprótesis traqueal y esofágica. El paciente continúa en seguimiento clínico.

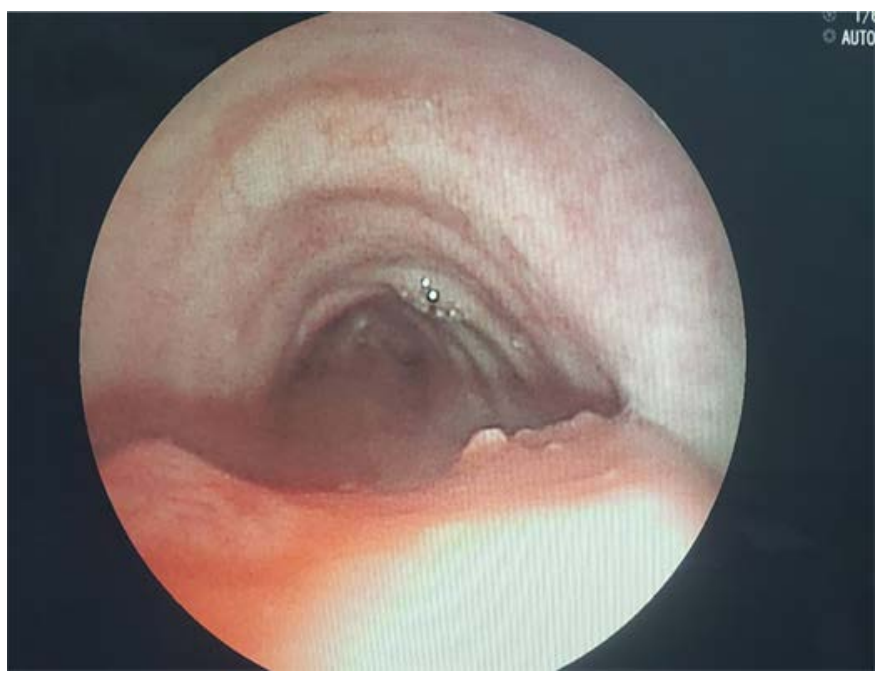

Figura 1. Aspecto broncoscópico de la fístula en la pared posterior de la tráquea donde se aprecia perdida de continuidad de la mucosa, edema y eritema. 


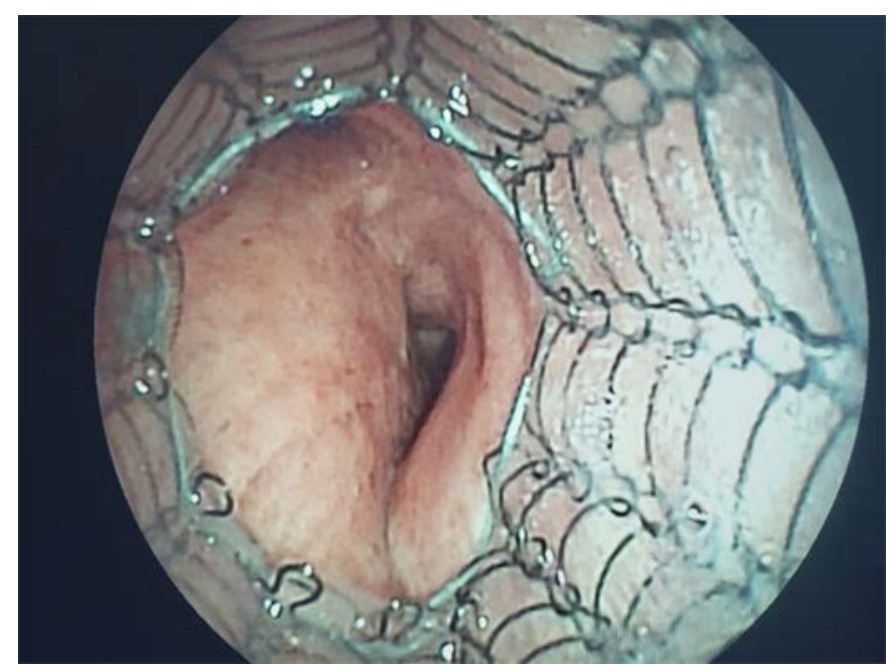

Figura 2. Imagen del stent ultraflex autoexpandible en posición adecuada dentro de la tráquea.

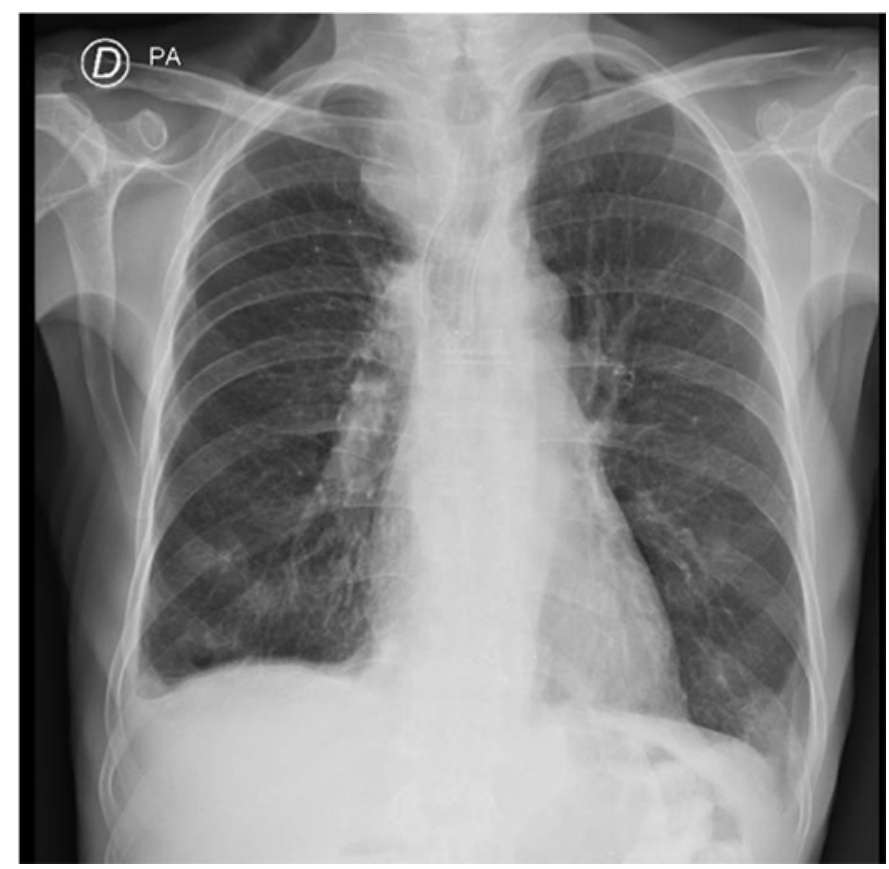

Figura 2. Radiografía de tórax donde se aprecian las prótesis traqueal y esofágica en posición

\section{Discusión}

Las FTE en adultos, son en su mayoría adquiridas. Su incidencia es difícil de establecer por la escasa literatura relacionada, así como por las dificultades en el diagnóstico. Sin embargo, pese a la baja frecuencia reportada, las FTE representan una alta tasa de morbimortalidad asociada (7). Pueden afectar esófago, pulmones y mediastino (8); son secundarias ya sea a procesos benignos, una minoría, o en la mayoría de los casos a entidades malignas que comprometen el mediastino (9).

Las FTE ocurren frecuentemente por invasión tumoral ya sea en esófago o pulmón o por complicaciones de terapias de cáncer como radioterapia, quimioterapia, tratamiento con láser, instrumentación o posterior a colocación de stent (4). La mayoría de las FTE se presentan en el tercio medio del esófago; el tercio distal corresponde a tumores de mayor tiempo de evolución y recaídas locales.

\section{Epidemiología}

Se considera que aproximadamente un 5-10\% de los pacientes con cáncer de esófago desarrollarán una FTE mientras un $1 \%$ corresponderá a carcinoma de pulmón $(2,5,10)$. Ballazs y colaboradores (10) reportaron que de 264 pacientes con FTE, un $92 \%$ presentó tumores esofágicos, $7 \%$ tumores pulmonares y $0.75 \%$ otros tumores de mediastino. Ciertos factores como desnutrición, infecciones asociadas, estado crítico y comorbilidades como diabetes mellitus, pueden agravar el proceso y aumentar la morbimortalidad (11). Se ha reportado un aumento del riesgo de FTE en los pacientes que utilizan terapias antiangiogénicas como Bevacizumab, especialmente en su combinación con radioterapia $(12,13)$.

\section{Diagnóstico}

La presentación clínica es inespecífica por lo que su diagnóstico se retrasa entre 1-18 meses desde las primeras manifestaciones clínicas (10).

Los síntomas más comunes son tos con la ingestión de líquidos o alimentos, bronquitis o neumonía purulenta recurrente y disfagia. Además, los pacientes se presentan con desnutrición proteicocalórica y aspiración recurrente (8). Si existe una historia de malignidad, se debe sospechar la presencia de una FTE. También se debe considerar el diagnóstico en pacientes con ventilación mecánica prolongada con síndrome de dificultad respiratoria del adulto, con trastorno de oxigenación asociado y distensión abdominal (10).

Las FTE pueden presentarse de manera aguda llevando el paciente a la muerte o subaguda presentando síntomas en días o semanas. En general, las FTE proximales de mayor tamaño tendrán síntomas más tempranos que las pequeñas distales. El diagnóstico de las FTE requiere la combinación de ayudas imagenológicas y endoscópicas, así como un alta sospecha 
clínica. Entre los estudios diagnósticos se debe incluir un esofagograma con bario que demuestre el paso de medio de contraste a la vía respiratoria estableciendo la presencia y el lugar de una fístula (14). Como alternativa o complemento al estudio de esofagograma en pacientes que no puedan deglutir, se podría considerar el uso de escanografía de tórax contrastada con reconstrucción tridimensional, que permita identificar áreas de necrosis tumoral por depósito de contraste en mediastino y además será de utilidad para la planificación del tratamiento posterior (15).

En la mayoría de los casos, posterior a las imágenes diagnósticas, se deben realizar estudios endoscópicos de vías digestivas y de vía respiratoria (fibrobroncoscopia) para confirmar y localizar la FTE. La localización, forma y tamaño de las fístulas puede variar en su presentación; las FTE pequeñas usualmente pasan desapercibidas porque la mucosa puede encontrarse con eritema y edema (4).

\section{Tratamiento}

Un enfoque multidisciplinario es necesario para determinar cuál es la mejor alternativa de tratamiento de acuerdo a cada paciente.

Los pacientes con FTE tanto por su patología oncológica como por tratamientos administrados, se presentan frecuentemente con deterioro clínico importante (7). Son pacientes que han recibido o van a recibir quimioterapia o radiación, presentan desnutrición y han tenido episodios de broncoaspiración recurrentes que predisponen a infecciones respiratorias severas con riesgo de falla respiratoria $(5,8)$. Por lo anterior, si bien se considera la intervención quirúrgica como primera opción terapéutica con una mayor sobrevida, se reserva está para pacientes candidatos a procedimientos quirúrgicos con enfermedad maligna localizada con intención curativa (16).

Existen por lo tanto otras opciones de menor invasividad, con adecuada efectividad, como las intervenciones endoscópicas que representan menor morbimortalidad comparada con la cirugía (17) y ayudan en el manejo sintomático con mejoría de la calidad de vida. Por lo tanto, el tratamiento para la mayoría de las FTE malignas será la colocación de stent esofágico, traqueal o doble stent, especialmente para FTE mayores a 5 $\mathrm{mm}$. Para aquellas de menos tamaño se podría considerar terapias broncoscópicas locales como electrocauterio o pegante tisular, entre otros (8).

\section{Stent de vía aérea}

La primera inserción de un stent metálico traqueal ocurre en el año 1952 (18). Desde entonces se han venido utilizando diferentes tipos de stent como alternativas para un cierre rápido y mínimamente invasivo de las FTE (8). Los stents rectos se utilizan a menudo para las FTE de las vías respiratorias proximales, mientras que los de forma en L o Y se reservan para compromiso traqueobronquial. Existen tres generaciones de stent, de los cuales los de primera generación ya no están en uso.

Los stent WallstentTM (Schneider Europe, Zurich, Suiza) de segunda generación son autoexpandibles una vez se liberan y los de tercera generación The UltraflexTM (Boston Scientific; Natick, MA, USA) utilizado en el caso reportado, son elaborados de una aleación de níquel-titanio con capacidad de "memoria de forma”, que significa que se deforma plásticamente a bajas temperaturas, volviendo a su forma inicial a altas temperaturas (19). Estos se insertan con broncoscopio flexible y sus ventajas son la expansibilidad y el ajuste fácil a las dimensiones traqueales, así como facilitan la eliminación de secreciones (20). Los problemas que conlleva son el crecimiento del tumor o tejido de granulación que hacen imposible su retiro, así como el riesgo de fractura con una incidencia del $12 \%$ después de largo tiempo (500-1.000 días) con restos metálicos que pueden generar complicaciones fatales (21).

Otros stent disponibles especialmente para casos de patología benigna son los stent siliconados. Fueron introducidos por Dumon en 1990 (22) para tratamiento de compresión extrínseca de la vía aérea; posteriormente fue usado por Weigert y colaboradores (23) en el manejo de las FTE malignas con resultados seguros y efectivos desde el punto de vista paliativo. Tienen como ventaja su facilidad para ser removidos y su mayor durabilidad. Para su colocación se necesita el fibrobroncoscopio rígido por lo que debe ser realizado en unidades con experiencia en su colocación. Son caracterizados por su flexibilidad y compatibilidad con tejido vecino. Sin embargo, tienen un riesgo de desplazamiento que se puede evitar con la fijación externa $y$ un incremento en el riesgo de infecciones ya que por su adherencia puede afectar el funcionamiento del sistema mucociliar (24). En un estudio retrospectivo se encontró que tanto los stent metálicos como los de silicona, tuvieron eficacia clínica equivalente, aunque la tasa de respuesta clínica completa fue levemente 
mayor con stent de silicona $(\mathrm{P}<0.05)(25)$.

Por especificaciones anatómicas, un stent esofágico puede predisponer a compresión de la vía aérea. Por lo que, para evitar esta complicación, en FTE maligna que afecta el tercio proximal y medio del esófago, se podría recomendar la colocación de doble stent $(8,26-$ 28), como se describió en nuestro paciente. Se debe precisar que el stent en la vía aérea debe ser colocado inicialmente. Esto reduce el riesgo de migración del stent esofágico y de obstrucción de vía aérea. Otras condiciones en las cuales se podría indicar colocación de doble stent serían: FTE inducida por stent esofágico, FTE mayores a $20 \mathrm{~mm}$ y en FTE sin estenosis esofágica evidente, puesto que el riesgo de migración posterior al stent esofágico es alto. Se debe considerar que el margen del stent esofágico debe quedar superior al stent de vía aérea para evitar desplazamiento y lograr el efecto terapéutico (25).

\section{Pronóstico}

Pese a la pésima condición clínica de los pacientes con FTE malignas, la colocación endoscópica de stent reporta tasas de mortalidad bajas del de $0 \%$ a $2 \%(10)$. Entre las complicaciones asociadas al procedimiento endoscópico se encuentra desplazamiento de stent, más frecuente con stent esofágico único. Con la colocación del doble stent se evita el desplazamiento, sin embargo, la presión concomitante de los dos stent podría producir reapertura de la FTE (5). Con relación a la tasa de supervivencia posterior a la colocación de stent, el estudio de Chen y colaboradores (29) demostró que el cierre de la FTE mejoraba sobrevida a corto plazo, comparado con gastrostomía/yeyunostomía. El estudio de Balazs y colaboradores (10) demostró una mejoría de la sobrevida hasta tres veces versus terapia de soporte y nutricional, evitando muertes tempranas por neumonía aspirativa y mejorando la calidad de vida. La mortalidad a largo plazo en pacientes con FTE tiene una estrecha relación a la patología oncológica, más no con la FTE.

\section{Conclusiones}

Las FTE malignas implican una pobre sobrevida en los pacientes con cáncer. Su tratamiento es un desafío clínico, debido a la condición general deteriorada de este tipo de pacientes. Se considera como manejo de primera línea la colocación de endoprótesis traqueal, esofágica o dual, con resultados óptimos. En nuestro caso se realizó un manejo paliativo con colocación de doble stent tanto en vía aérea como esofágica, para evitar obstrucción de vía aérea, mejorar sintomatología y calidad de vida. Se continúa en búsqueda de estrategias terapéuticas que puedan aumentar la sobrevida y repercutan en la calidad de vida de los pacientes con cáncer.

\section{Referencias}

1. Martini N, Goodner JT, D'Angio GJ, Beattie EJ. Tracheoesophageal fistula due to cancer. J Thorac Cardiovasc Surg. 1970 Mar;59(3):319-24.

2. Morgan RA, Ellul JP, Denton ER, Glynos M, Mason RC, Adam A. Malignant esophageal fistulas and perforations: management with plastic-covered metallic endoprostheses. Radiology. 1997 Aug;204(2):527-32. doi: 10.1148/radiology.204.2.9240548.

3. Shamji FM, Inculet R. Management of Malignant Tracheoesophageal Fistula. Thorac Surg Clin. 2018 Aug;28(3):393-402. doi: 10.1016/j.thorsurg.2018.04.007.

4. Kim KR, Shin JH, Song HY, Ko GY, Kim JH, Yoon HK, et al. Palliative treatment of malignant esophagopulmonary fistulas with covered expandable metallic stents. AJR Am J Roentgenol. 2009 Oct;193(4):W278-82. doi: 10.2214/AJR.08.2176.

5. Hürtgen M, Herber SCA. Treatment of malignant tracheoesophageal fistula. Thorac Surg Clin. 2014 Feb;24(1):117-127. doi: 10.1016/j.thorsurg.2013.09.006.

6. Aworanti O, Awadalla S. Management of recurrent tracheoesophageal fistulas: a systematic review. Eur J Pediatr Surg. 2014 Oct;24(5):365-75. doi: 10.1055/s-0034-1370780.

7. Qureshi YA, Muntzer Mughal M, Fragkos KC, Lawrence D, George J, Mohammadi B, Dawas $\mathrm{K}$, et al. Acquired Adult Aerodigestive Fistula: Classification and Management. J Gastrointest Surg. 2018 Oct;22(10):1785-1794. doi: 10.1007/ s11605-018-3811-0.

8. Zhou C, Hu Y, Xiao Y, Yin W. Current treatment of tracheoesophageal fistula. Ther Adv Respir Dis. 2017 Apr;11(4):173-180. doi: 10.1177/1753465816687518.

9. Couraud L, Ballester MJ, Delaisement C. Acquired tracheoesophageal fistula and its management. Semin Thorac Cardiovasc Surg. 1996 Oct;8(4):392-9. 
10. Balazs A, Kupcsulik PK, Galambos Z. Esophagorespiratory fistulas of tumorous origin. Non-operative management of 264 cases in a 20-year period. Eur J Cardiothorac Surg. 2008 Nov;34(5):1103-7. doi: 10.1016/j. ejcts.2008.06.025.

11. Chakraborty RK, Hoag JB. Acquired Aero Digestive Fistula in Adults-Case Series and Review. EC Pulmonol Respir Med. 2019;8(2):123-139. Disponible en: https://www.ecronicon.com/ ecprm/pdf/ECPRM-08-00337.pdf

12. Goodgame B, Veeramachaneni N, Patterson A, Govindan R. Tracheo-esophageal fistula with bevacizumab after mediastinal radiation. J Thorac Oncol. 2008 Sep;3(9):1080-1. doi: 10.1097/JTO. 0b013e3181858eba.

13. Gore E, Currey A, Choong N. Tracheoesophageal fistula associated with bevacizumab 21 months after completion of radiation therapy. J Thorac Oncol. 2009 Dec;4(12):1590-1. doi: 10.1097/ JTO.0b013e3181c06a6f.

14. Gore RM, Levine M. Textbook of Gastrointestinal Radiology, 2nd ed, W. B. Saunders Company, Philadelphia, PA. 2000.

15. Wang H, Tao M, Zhang N, Li D, Zou H, Zhang J, et al. Airway Covered Metallic Stent Based on Different Fistula Location and Size in Malignant Tracheoesophageal Fistula. Am J Med Sci. 2015 Nov;350(5):364-8. doi: 10.1097/ MAJ.0000000000000565.

16. Lenz CJ, Bick BL, Katzka D, Nichols FC, Depew ZS, Wong Kee Song LM, et al. Esophagorespiratory Fistulas: Survival and Outcomes of Treatment. J Clin Gastroenterol. 2018 Feb;52(2):131136. doi: 10.1097/MCG.0000000000000751.

17. Meier JD, Sulman CG, Almond PS, Holinger LD. Endoscopic management of recurrent congenital tracheoesophageal fistula: a review of techniques and results. Int J Pediatr Otorhinolaryngol. 2007 May;71(5):691-7. doi: 10.1016/j. ijporl.2007.02.022.

18. Harkins WB. An endotracheal metallic prosthesis in the treatment of stenosis of the upper trachea. Ann Otol Rhinol Laryngol. 1952 Sep;61(3):66376. doi: 10.1177/000348945206100307.

19. Vinograd I, Klin B, Brosh T, Weinberg M, Flomenblit $Y$, Nevo Z. A new intratracheal stent made from nitinol, an alloy with "shape memory effect". J Thorac Cardiovasc Surg. 1994 May;107(5):1255-61.

20. Madden BP, Datta S, Charokopos N. Experien- ce with Ultraflex expandable metallic stents in the management of endobronchial pathology. Ann Thorac Surg. 2002 Mar;73(3):938-44. doi: 10.1016/s0003-4975(01)03460-9.

21. Chung FT, Lin SM, Chen HC, Chou CL, Yu CT, Liu CY, Wang CH, Lin HC, Huang CD, Kuo HP. Factors leading to tracheobronchial self-expandable metallic stent fracture. J Thorac Cardiovasc Surg. 2008 Nov;136(5):1328-35. doi: 10.1016/j. jtcvs.2008.05.039.

22. Dumon JF. A dedicated tracheobronchial stent. Chest. 1990 Feb;97(2):328-32. doi: 10.1378/ chest.97.2.328.

23. Weigert $\mathrm{N}$, Neuhaus $H$, Rösch T, Hoffmann W, Dittler HJ, Classen M. Treatment of esophagorespiratory fistulas with silicone-coated self-expanding metal stents. Gastrointest Endosc. 1995 May;41(5):490-6. doi: 10.1016/s00165107(05)80009-4.

24. Mitsuoka M, Sakuragi T, Itoh T. Clinical benefits, and complications of Dumon stent insertion for the treatment of severe central airway stenosis or airway fistula. Gen Thorac Cardiovasc Surg. 2007 Jul;55(7):275-80. doi: 10.1007/s11748-0070131-9.

25. Ke M, Wu X, Zeng J. The treatment strategy for tracheoesophageal fistula. J Thorac Dis. 2015 Dec; 7(Suppl 4):S389-97. doi: 10.3978/j.issn.20721439.2015.12.11.

26. Bick BL, Song LM, Buttar NS, Baron TH, Nichols FC, Maldonado F, et al. Stent-associated esophagorespiratory fistulas: incidence and risk factors. Gastrointest Endosc. 2013 Feb;77(2):181-9. doi: 10.1016/j.gie.2012.10.004.

27. Yamamoto R, Tada H, Kishi A, Tojo T, Asada $H$. Double stent for malignant combined esophago-airway lesions. Jpn J Thorac Cardiovasc Surg. 2002 Jan;50(1):1-5. doi: 10.1007/BF02913479.

28. Herth FJ, Peter S, Baty F, Eberhardt R, Leuppi JD, Chhajed PN. Combined airway and oesophageal stenting in malignant airway-oesophageal fistulas: a prospective study. Eur Respir J. 2010 Dec;36(6):1370-4. doi: 10.1183/09031936.00049809.

29. Chen YH, Li SH, Chiu YC, Lu HI, Huang CH, Rau KM, et al. Comparative study of esophageal stent and feeding gastrostomy/jejunostomy for tracheoesophageal fistula caused by esophageal squamous cell carcinoma. PLoS One. 2012;7(8):e42766. doi: 10.1371/journal. pone.0042766. 